\title{
Tocilizumab monotherapy and adalimumab monotherapy go head-to-head in RA
}

A phase IV superiority study in patients with severe rheumatoid arthritis (RA) receiving tocilizumab or adalimumab as monotherapy (without concomitant methotrexate) has shown that tocilizumab is superior to adalimumab for reducing the severity of disease in this setting, as now published in The Lancet.

\section{4 ...the first study to specifically assess the superiority of biologic agents from different classes as monotherapies... 77}

Although many patients with RA take a biologic agent in addition to background therapy with methotrexate or another nonbiologic DMARD, approximately one third take a biologic agent as monotherapy. Nevertheless, comparative data are lacking. This randomized, double-blind, parallel-group trial (sponsored by F Hoffman-La Roche), is the first study to specifically assess the superiority of biologic agents from different classes as monotherapies -the trial drugs, tocilizumab and adalimumab, are fully human monoclonal antibodies that target IL-6 and TNF signalling, respectively.

Adults with severe RA for $>6$ months duration and who could not take methotrexate were randomly assigned to receive tocilizumab $(8 \mathrm{mg} / \mathrm{kg}$ intravenously every 4 weeks, plus subcutaneous placebo every 2 weeks; $n=163$ ) or adalimumab (40 mg subcutaneously every 2 weeks plus intravenous placebo every 4 weeks; $n=162$ ) for 24 weeks. The primary outcome was change in the disease activity score using 28 joints (DAS28) from baseline to 24 weeks.

$15 \%$ of patients in the tocilizumab group and $17 \%$ in the adalimumab group withdrew from the study. Safety reasons for discontinuation included adverse events ( 9 for tocilizumab; 10 for adalimumab) and two people in the tocilizumab group died. The mean change in DAS28 from baseline to week 24 was greater in the tocilizumab group than in the adalimumab group: -3.3 compared with -1.8 (difference of $-1.5,95 \%$ CI -1.8 to $-1.1 ; P<0.0001)$. Serious adverse events were more frequent in the tocilizumab group than the adalimumab group (12\% versus $10 \%$ ) and more patients receiving tocilizumab had increased LDL-cholesterol, increased alanine aminotransferase levels, and reduced platelet or neutrophil counts than patients receiving adalimumab.

Jenny Buckland

Original article Gabay, C. et al. Tocilizumab monotherapy versus adalimumab monotherapy for treatment of rheumatoid arthritis (ADACTA): a randomised, doubleblind, controlled phase 4 trial. Lancet doi:10.1016/ s0140-6736(13)60250-0 